\title{
Expression levels of Fas/Fas-L mRNA in human brain glioma stem cells
}

\author{
JUN TAO, BO QIU, DONGYONG ZHANG and YUNJIE WANG \\ Department of Neurosurgery, First Hospital of China Medical University, Shenyang, Liaoning 110001, P.R. China
}

Received November 13, 2011; Accepted February 6, 2012

DOI: $10.3892 / \mathrm{mmr} .2012 .791$

\begin{abstract}
Despite advancements in research made over the past decades, the prognosis of gliomas remains dismal. Fas/ Fas ligand (Fas-L)-related immunotherapy may be regarded as a treatment of choice as it can induce the apoptosis of glioma cells, and has shown promising results in experimental studies. Over the years, brain glioma stem cells (BGSCs) have been accepted as the origin of gliomas and determine their biological features. The theory of BGSCs has facilitated the study of gliomas. In this study, we conducted a series of assays to culture BGSCs from clinical samples and determined the mRNA expression levels of Fas/Fas-L in BGSCs. We also investigated the effects of Fas/Fas-L-related immunotherapy on the apoptosis of glioma cells. BGSCs were grown from samples of 8 patients suffering from gliomas and identified by the assessment of biological characteristics and immunocytochemistry. Total RNA was extracted and reverse-transcribed into cDNA, and the expression levels of Fas/Fas-L mRNA were determined by real-time RT-PCR, followed by statistical analysis. The results showed that the Fas and Fas-L mRNA expression levels in BGSCs were lower compared to those in primary cultured glioma cells, which were statistically significant $(\mathrm{P}<0.001)$. These results indicate that immunotherapy involving Fas/Fas-L may not eradicate the BGSCs, which would result in the relapse of glioma. However, further research is required to investigate the mechanisms involved and define the prospect of Fas-involved immunotherapy against gliomas.
\end{abstract}

\section{Introduction}

As the most frequent brain tumors, gliomas, especially highgrade gliomas (malignant gliomas, e.g., glioblastoma multiforme and anaplastic astrocytomas), represent the poorest prognosis among primary intracranial tumors. Despite recent

Correspondence to: Dr Jun Tao, Department of Neurosurgery, First Hospital of China Medical University, No. 155 North Nanjing Street, Heping, Shenyang, Liaoning 110001, P.R. China

E-mail: cmu_taojun@yahoo.com

Key words: gliomas, brain glioma stem cells, Fas/Fas-L, immunotherapy advances made in surgery, radiotherapy and chemotherapy, the morbidity of high-grade brain gliomas is almost unchangeable, and remains one of the toughest challenges for neurosurgeons. The biological features of brain gliomas, such as extensively infiltrative and invasive growth, result in resistance to radical surgical therapy, radiotherapy and chemotherapy. Many studies have been carried out to improve the outcome of these therapies. Apoptosis mediated by Fas and Fas Ligand (Fas/Fas-L) is constantly a major focus of studies on glioma treatment (1). Moreover, the theory of brain glioma stem cells (BGSCs) and has provided more aspects and opportunities to better understand gliomas (2-5). Thus, BGSCs were grown from clinical samples of high-grade brain glioma patients, the mRNA expression levels of Fas/Fas-L were determined, and their effects on the apoptosis of glioma cells and Fas-related immunotherapies were estimated, which may help to provide further knowledge on the biological characteristics of malignant brain gliomas.

\section{Materials and methods}

Glioma specimens collection. All samples were obtained from the resected glioma tissues from 8 patients at the Neurosurgery Department, as approved by the Institutional Review Boards of the First Clinical Hospital, China Medical University. Tumors were graded at the China Medical University by the experienced neuropathologists in accordance with the WHO established guidelines. Seventeen samples were included, of which 8 samples successfully grew BGSCs. In 8 gliomas, there were 6 anaplastic gliomas (grade III) and 2 glioblastoma multiformes (grade IV), including 3 males and 5 females; the ages varied from 44 to 75 years (mean, 53.75).

Primary culture, cell propagation and cloning. Primary glioma cells and tumor cell sphere cultures were performed as previously described [Singh et al (2-3)] (4,5), with some modifications. Briefly, the samples were preserved at $4^{\circ} \mathrm{C}$ after resection, and managed in $30 \mathrm{~min}$. Tissues were washed, minced, digested enzymatically, dissociated and passed through a series of cell strainers. Cells were seeded in DMEM/ F12 medium (Gibco) supplemented with recombined human basic fibroblast growth factor (rh-bFGF; $20 \mathrm{ng} / \mathrm{ml}$, Invitrogen), recombined human epidermal growth factor (rh-EGF; $20 \mathrm{ng}$ / ml, Invitrogen), B-27 supplement (1:50, without serum and vitamin A; Gibco-Invitrogen), $100 \mathrm{IU} / \mathrm{ml}$ penicillin $\mathrm{G}$ and $100 \mu \mathrm{g} / \mathrm{ml}$ streptomycin, at a density of $2 \times 10^{5}$ live cells $/ \mathrm{ml}$. 
Primary glioma cells were grown in DMEM/F12 medium with $15 \%$ fetal bovine serum (FBS; Gibco), and passaged over 4 generations. When tumor cell sphere formation was noted, they were dissociated into single cells by mechanical trituration through a fire-polished Pasteur pipette, and reseeded into the same stem cell medium at the same density.

Limiting dilution assays. The tumor spheres of each sample were passaged over 4 generations. Tumor spheres of 3-4 generations were collected, dissociated and plated in 96-well microwell plates in $0.2 \mathrm{ml}$ volumes of stem cell medium (DMEM/F-12 with rh-bFGF, rh-EGF and B-27). After serial dilution, the final cell density was $1-2$ cells/well. Cultures were fed $0.025 \mathrm{ml}$ of stem cell medium every 2 days. On day 7, each well was observed under a microscope to evaluate the clonal formation ability of tumor sphere cells.

Differentiation assay of tumor spheres. Tumor spheres of 3-4 generations were harvested and moved onto anti-peeling slides pre-coated with poly-L-lysine (Sigma) in DMEM/F-12 with $15 \%$ FBS and B-27, but without rh-bFGF and rh-EGF. The spheres were inspected under a microscope at regular intervals. Seven days later, the tumor spheres after induced differentiation were subjected to immunocytochemistry assay.

Immunocytochemistry assays. Undifferentiated tumor spheres of 3-4 generations were collected, plated onto anti-peeling slides and cultured in stem cell medium for $4 \mathrm{~h}$. Tumor spheres were then fixed with $4 \%$ paraformaldehyde and stained with antibodies against CD133 (mouse monoclonal antibodies, 1:200; Abcam) at $4^{\circ} \mathrm{C}$, followed by $\mathrm{Cy} 3$-conjugated goat anti-mouse secondary antibody (1:50; Sigma). Nuclei were counterstained with 4,6-diamidino-2-phenylindole (DAPI).

Tumor spheres after induced differentiation were fixed with 4\% paraformaldehyde and stained with antibodies against glial fibrillary acidic protein (GFAP; monoclonal rabbit anti-GFAP, 1:200; Bioworld) for glial cells and TU-20 (monoclonal mouse anti- $\beta$-tubulin III isoform, C-terminus, 1:200; Millipore) for neurons, for $24 \mathrm{~h}$ at $4^{\circ} \mathrm{C}$. Cy3- and FITC-conjugated secondary antibodies (goat anti-rabbit and goat anti-mouse, 1:50; Sigma) were used, respectively. Nuclei were counterstained with DAPI. All stained cells were finally observed under an Olympus BX61 fluorescent microscope and imaged.

Extraction of total RNA and real-time quantitative RT-PCR. In brief, the cultured primary glioma cells and tumor spheres (3-4 passage) were collected and homogenized in TRIzol (Takara), followed by total RNA extraction with an RNAsimple Total RNA kit (Tiangen Biotech). mRNA expression levels of Fas/Fas-L were detected by real-time RT-PCR. The reaction was performed on an ABI PRISM 7000 Real-Time PCR System (Applied Biosystems), using an SYBR PrimeScript RT-PCR Two-Step kit (Takara) according to the manufacturer's instructions, and GAPDH was used as the internal reference. The reaction volume was $20 \mu 1$ for each gene. All primers were designed and synthesized by Takara (Table I). The PCR condition was $95^{\circ} \mathrm{C}$ for $10 \mathrm{sec}$, then $95^{\circ} \mathrm{C}$ for $5 \mathrm{sec}$ and $60^{\circ} \mathrm{C}$ for $31 \mathrm{sec}, 40$ cycles. The quantitative RT-PCR data were assessed using $\Delta \Delta \mathrm{CT}$ for evaluating the results with the ABI Prism 7000 Software. A standard melting-curve cycle
Table I. Primer details.

\begin{tabular}{ll}
\hline Fas (F) & 5' TTC TGC CAT AAG CCC TGT CC 3' \\
Fas (R) & 5' TGT ACT CCT TCC CTT CTT GG 3' \\
Fas-L (F) & 5' GCC TGT GTC TCC TTG TGA TG 3' \\
Fas-L (R) & 5' TGG ACT TGC CTG TTA AAT GGG 3' \\
GAPDH (F) & 5' GCACCGTCAAGGCTGAGAAC 3' \\
GAPDH (R) & 5' TGGTGAAGACGCCAGTGGA 3' \\
\hline
\end{tabular}

F, forward; R, reverse.

was used to examine the quality of amplification. For each analysis, samples were normalized by comparison with the housekeeping gene, GAPDH.

Statistical analysis. In real-time RT-PCR, data were analyzed by the two-tailed paired t-test and presented as the means \pm standard deviation with SigmaPlot 11.0 software (Systat Software). The significance level was set at 0.05 . The results were visualized with histograms.

\section{Results}

Brain glioma cells form tumor spheres and can be serially passaged. After dissociation and primary culture, the glioma cells were grown as monolayers in medium containing FBS, then they were switched to medium permissive for stem cell proliferation. Within 48-72 h, single cell division could be observed, and 5-7 days later a large amount of neurospherelike clusters was formed. These tumor spheres were suspended and were round or oval appearance, each comprised 4-10 cells, with fine refraction under a light microscope. Some tumor spheres showed irregular aspects. The majority of monolayer cells remained adherent and continued to differentiate, which were removed during passage. Tumor spheres enlarged their volumes and cell amounts after multiple passages, meanwhile, the generation time was shortened. After dissociation and reseeding in the stem cell medium, the tumor sphere cells formed new subspheres. Images of the tumor spheres are shown in Fig. 1.

Tumor sphere cells show ability of self-renewal. The serial passage of tumor sphere cells showed that they have favorable proliferative ability. Some tumor spheres were harvested and subjected to subsphere formation by limiting dilution. After dissociation, tumor cells were seeded in microwell plates at a density of 1-2 cells/well. Seven days later, secondary subspheres were observed under a microscope. These one mother cellderived spheres were dissociated into individual cells and reseeded as described above. New secondary subspheres from single cells were formed again. Limiting dilution assay showed the self-renewal ability of the tumor sphere cells.

Immunocytochemistry of tumor spheres and their differentiated progeny. As a cell surface marker of normal human neural stem cells, CD133 has been generally used for identifying BGSCs (2-4). Tumor spheres fixed on slides were stained and inspected under a fluorescent microscope. The undifferenti- 


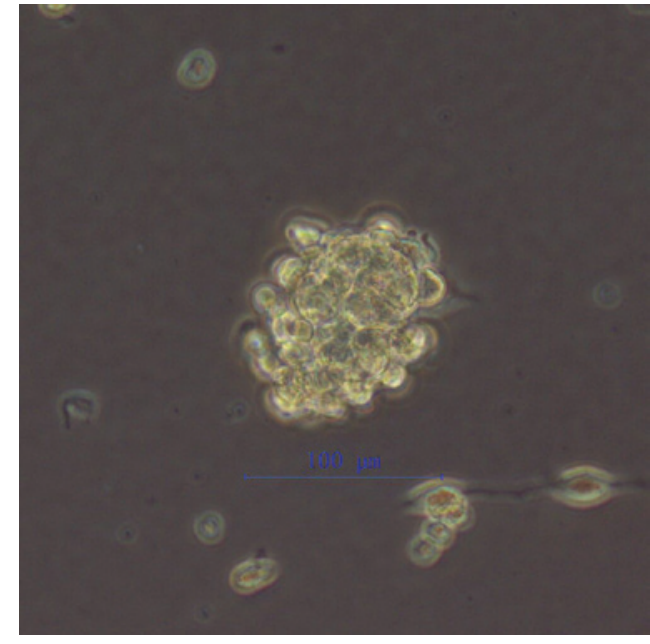

Figure 1. Tumor cell spheres cultured in committed stem cell medium (magnification, $\mathrm{x} 200$ ).

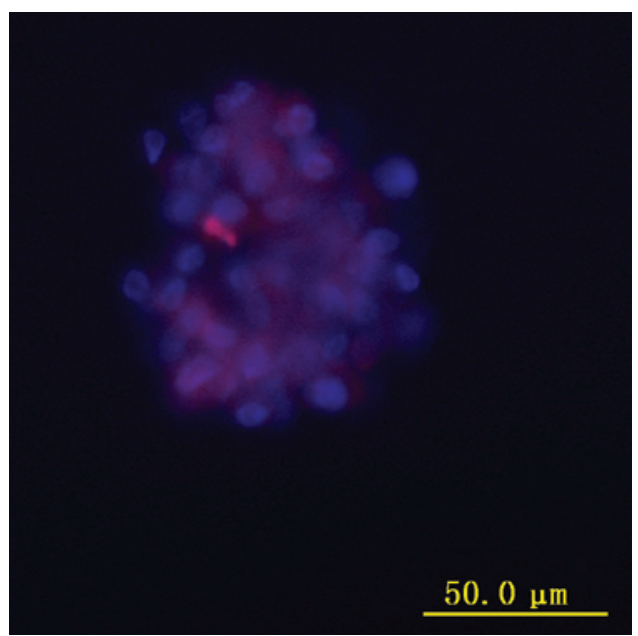

Figure 2. Immunocytochemistry showed that the tumor cell spheres were CD133-positive (red), and the nuclei were stained with DAPI (blue).

ated tumor spheres exhibited immunoreactivity for CD133, as shown in Fig. 2.

After induced differentiation assay, the differentiated tumor sphere cells were subjected to immunostaining of GFAP and TU-20. The results proved that these cells expressed markers of at least two kinds of mature neural cells, glial cells (GFAP) and neurons (TU-20), as shown in Fig. 3. Double-positive staining indicated that these tumor sphere cells were multipotent.

Fas/Fas-L mRNA expression levels are higher in tumor sphere cells. Real-time RT-PCR detection was conducted in the tumor sphere cells and mature primary glioma cells as described above. A certain amount of mRNA up to the requirement was extracted from each sample and reverse-transcribed into cDNA, followed by PCR. The $\Delta \Delta C T$ method was used to evaluate the relative expression quantity of Fas/Fas-L. The results revealed that the mRNA expression levels of tumor sphere cells were higher than those of differentiated primary glioma cells. One-way ANOVA analysis was performed to calculate the means \pm standard deviation with SigmaPlot 11.0 software, followed by a two-tailed t-test, which showed $\mathrm{p}<0.001$ for Fas/
A

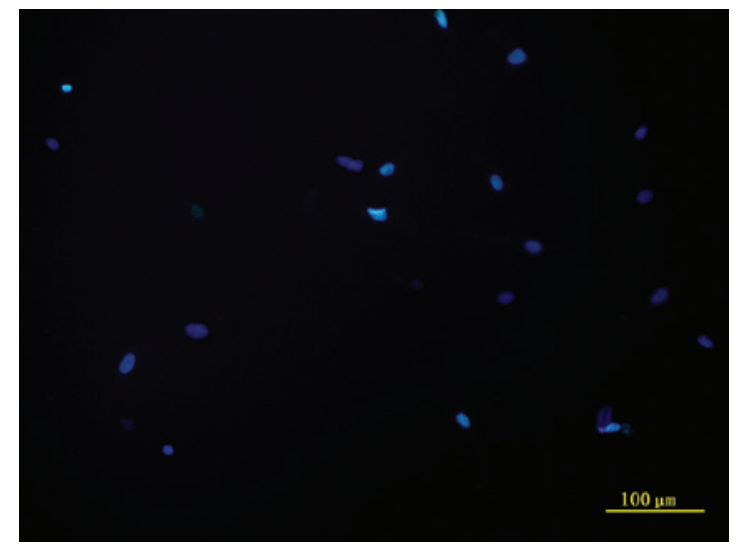

B

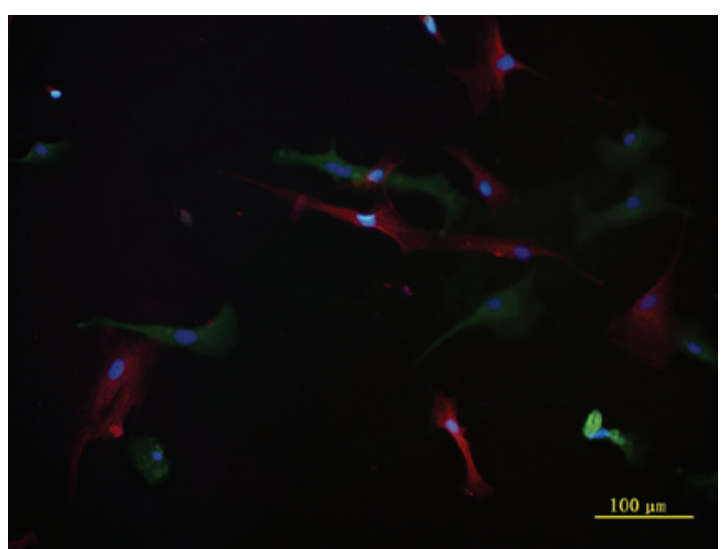

Figure 3. After induced differentiation, immunochemistry showed that the differentiated tumor cell spheres express GFAP (cy3 as the secondary antibody, red), and TU-20 (FITC as the secondary antibody, green). The nuclei were counterstained with DAPI. The induced differentiation assay proved the multipotency of tumor cell spheres.

Fas-L in both groups, with significant statistical difference. The results were converted to a histogram, as shown in Fig. 4.

\section{Discussion}

Brain gliomas comprise 33.3-58.9\% of intracranial tumors, and are the most intractable among primary brain tumors. Over the years, the incidence of gliomas is still rising, but the prognosis has not improved (6). Scientists have made great efforts to investigate treatments for brain gliomas; inducing the apoptosis of glioma cells is one of the treatment options. Fas/Fas-L interaction induces the assembly of the deathinducing signaling complex, including Fas, Fas-associated death domain (FADD) and caspases, and culminates in the apoptosis of Fas-positive cells. Fas is not expressed in normal brain tissues, but glioma cells are Fas-positive, which provides a promising therapeutic strategy for gliomas.

Tachibana et al reported a correlation between Fas expression and the malignancy grade of astrocytomas, indicating the impact of Fas on glioma progression (7). Frei et al documented that malignant glioma cells are sensitive to Fas-L-mediated apoptosis ex vivo (8). Roth et al demonstrated that Fas-L can synergistically induce the apoptosis of Fas-positive glioma cell lines accompanied with chemotherapeutic agents, and the sensitivity of Fas-L-mediated apoptosis is related to Fas expression on the cell surface (1). All the findings above indicate that Fas induces cell apoptosis by binding Fas antibodies 
A
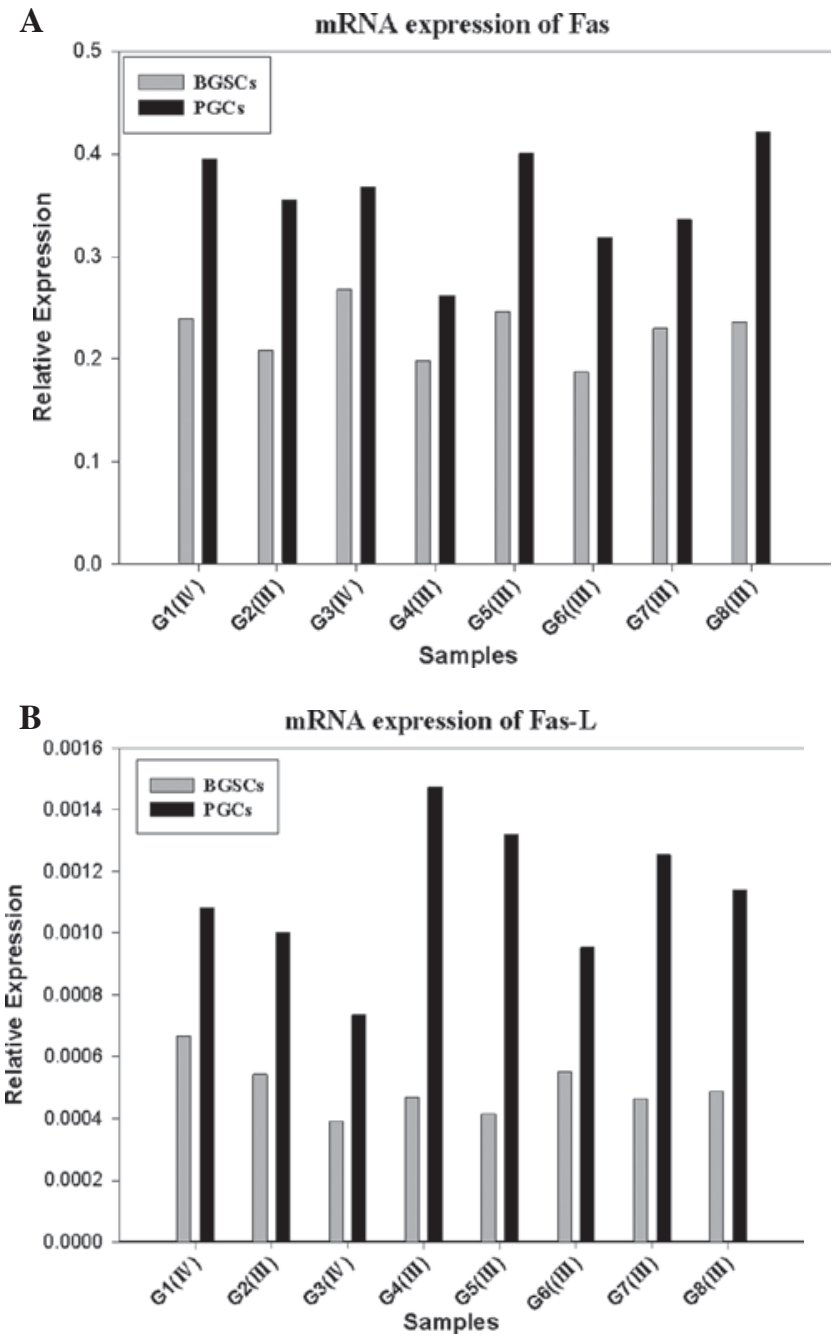

Figure 4. After real-time RT-PCR determination, a paired t-test revealed that the mRNA expression of both Fas/Fas-L in BGSCs was lower compared to their counterparts in primary glioma cells, and the differences were statistically significant ( $\mathrm{p}<0.001$ for both factors).

or Fas-L, which is important for glioma immunotherapy. Chen et al confirmed that Fas-L is expressed in most gliomas and is negative in normal brain tissues, but there is no evident correlation between Fas-L expression and glioma malignancy; besides, Fas protein expression levels increase when the malignant degree of gliomas is elevated (9). Although many glioma immunotherapies targeting Fas are still empirical studies, certain experimental results have shown a promising prospect for glioma immunotherapies $(10,11)$.

Over the years, the finding of BGSCs is an important advance in glioma studies. BGSCs have proven to be the 'initiating cells' of gliomas, which are pivotal in glioma initiation, progression and recurrence. In addition, CD133 is the most preferred marker for identifying BGSCs (2-4). Though the mechanisms behind BGSCs remain unknown, the theory of BGSCs and relevant research results have shown that BGSCs may become a new target for the treatment of gliomas. At present, it is generally accepted that gliomas cannot be cured unless all the BGSCs are eradicated, as new tumors will inevitably form once any BGSCs survive the glioma therapies $(12,13)$.
According to the theories described above, we speculate that any immunotherapies targeting Fas take effect and eliminate gliomas only if they can eradicate the BGSCs. Several previous investigations on the biological characteristics of BGSCs have demonstrated that BGSCs are resistant to multiple therapeutic strategies, including radiotherapy and chemotherapy, which leads to the failure to cure gliomas; moreover, the gliomas will recur inevitably if BGSCs survive any therapy, as the residual BGSCs initiate tumorigenesis and form new glioma foci $(14,15)$. In addition, immunosuppression and immune escape are also difficulties in the immunotherapy of gliomas $(16,17)$. Therefore, the efficacy and prospect of immunotherapies targeting Fas/Fas-L still need to be proven by further studies.

In this study, we successfully grew BGSCs from clinical glioma specimens, and determined mRNA expression of Fas/ Fas-L by quantitative real-time RT-PCR. The results showed that either Fas or Fas-L mRNA expression in BGSCs was lower than its corresponding differentiated primary glioma cells. It is known that immunotherapies based on Fas, regardless of Fas antibodies or Fas-L, are all targeting Fas. Weller et al reported that gliomas would resist Fas antibody-mediated apoptosis if Fas expression is low or undetectable (18). In this study, we detected low mRNA expression levels of Fas and Fas-L in BGSCs. From these results, it can perhaps be concluded that immunotherapies targeting Fas are not very effective, which is not very optimistic for the treatment of gliomas. However, only the mRNA expression levels were detected and, as we know, gene expression abundance is a key issue and determines the practical effects of Fas/Fas-L. Moreover, signal transduction intensity is also important for the final effects of Fas/ Fas-L. Nevertheless, low expression of Fas/Fas-L in BGSCs is consistent with other biological characteristics of BGSCs; the tendency of immortalization (escape from apoptosis) and resistance to other treatments, which may exert a negative impact on various therapies targeting Fas.

In this study, we sought to determine the mRNA expression of Fas/Fas-L in BGSCs, and estimate their effects on glioma immunotherapies targeting Fas. We anticipate that low expression of Fas/Fas-L in BGSCs may lead to the failure of immunotherapies targeting Fas, since the BGSCs could not be eradicated and may result in the recurrence of gliomas. A study by Bertrand et al also showed that human glioma stem cells from the U87 cell line were resistant to Fas-induced apoptosis (19), which is consistent with our results. The theory of BGSCs has practically altered our cognition of gliomas in various aspects, and has challenged many current therapeutic strategies of gliomas, such as the selection of therapeutic targets and glioma immunotherapies $(12,13,20,21)$. In fact, to elucidate the biological features of glioma simply based on any individual gene is difficult, since glioma initiation, progression and relapse are complex processes, and the expression of various genes and related immunoregulation and the signaling pathways involved in immunotherapies are complicated. The experimental result that mRNA expression levels of Fas/Fas-L are lower in BGSCs is just one of multiple biological characteristics of gliomas. Further investigations are warranted to better evaluate the effects of BGSCs in the biological processes of gliomas, and to more accurately understand the prospect of Fas/Fas-L in glioma treatments. 


\section{Acknowledgements}

This study was supported by the Foundation of Liaoning Provincial Commission of Science Technology (No. 2007408001-5).

\section{References}

1. Roth W, Fontana A, Trepel M, Reed JC, Dichgans J and Weller M Immunochemotherapy of malignant glioma: synergistic activity of CD95 ligand and chemotherapeutics. Cancer Immunol Immunother 44: 55-63, 1997.

2. Singh SK, Clarke ID, Terasaki M, et al: Identification of a cancer stem cell in human brain tumors. Cancer Res 63: 5821-5828, 2003

3. Singh S and Dirks PB: Brain tumor stem cells: identification and concepts. Neurosurg Clin N Am 18: 31-38, 2007.

4. Galli R, Binda E, Orfanelli U, et al: Isolation and characterization of tumorigenic, stem-like neural precursors from human glioblastoma. Cancer Res 64: 7011-7021, 2004.

5. Yuan X, Curtin J, Xiong Y, et al: Isolation of cancer stem cells from adult glioblastoma multiforme. Oncogene 23: 9392-9400, 2004.

6. Hess KR, Broglio KR and Bondy ML: Adult glioma incidence trends in the United States, 1977-2000. Cancer 101: 2293-2299, 2004.

7. Tachibana O, Nakazawa H, Lampe J, Watanabe K, Kleihues $P$ and Ohgaki H: Expression of Fas/APO-1 during the progression of astrocytomas. Cancer Res 55: 5528-5530, 1995.

8. Frei K, Ambar B, Adachi N, Yonekawa Y and Fontana A: Ex vivo malignant glioma cells are sensitive to Fas (CD95/APO-1) ligandmediated apoptosis. J Neuroimmunol 87: 105-113, 1998.

9. Chen Y, Zhang X, Bu X, Wang Z and Zhang X: The expression of Fas and Fas Ligand in human gliomas. J Fourth Mil Med Univ 21: 80-82, 2000.

10. Ho IA, Hui KM and Lam PY: Targeting proliferating tumor cells via the transcriptional control of therapeutic genes. Cancer Gene Ther 13: 44-52, 2006.
11. Giraud S, Bessette B, Boda C, et al: In vitro apoptotic induction of human glioblastoma cells by Fas ligand plus etoposide and in vivo antitumour activity of combined drugs in xenografted nude rats. Int J Oncol 30: 273-281, 2007.

12. Behbod F and Rosen JM: Will cancer stem cells provide new therapeutic targets? Carcinogenesis 26: 703-711, 2005.

13. Tunici P, Irvin D, Liu G, et al: Brain tumor stem cells: new targets for clinical treatments? Neurosurg Focus 20: 27, 2006

14. Bao S, Wu Q, McLendon RE, et al: Glioma stem cells promote radioresistance by preferential activation of the DNA damage response. Nature 444: 756-760, 2006.

15. Liu G, Yuan X, Zeng Z, et al: Analysis of gene expression and chemoresistance of $\mathrm{CD} 133^{+}$cancer stem cells in glioblastoma. Mol Cancer 5: 67, 2006.

16. Gomez GG and Kruse CA: Mechanisms of malignant glioma immune resistance and sources of immunosuppression. Gene Ther Mol Biol 10: 133-146, 2006.

17. Kennedy BC, Maier LM, D'Amico R, et al: Dynamics of central and peripheral immunomodulation in a murine glioma model. BMC Immunol 10: 11, 2009.

18. Weller M, Frei K, Groscurth P, Krammer PH, Yonekawa Y and Fontana A: Anti-Fas/APO-1 antibody-mediated apoptosis of cultured human glioma cells. Induction and modulation of sensitivity by cytokines. J Clin Invest 94: 954-964, 1994.

19. Bertrand J, Begaud-Grimaud G, Bessette B, Verdier M, Battu S and Jauberteau MO: Cancer stem cells from human glioma cell line are resistant to Fas-induced apoptosis. Int J Oncol 34: 717-727, 2009.

20. Wei J, Barr J, Kong LY, et al: Glioma-associated cancer-initiating cells induce immunosuppression. Clin Cancer Res 16: 461-473, 2010.

21. Di Tomaso T, Mazzoleni S, Wang E, et al: Immunobiological characterization of cancer stem cells isolated from glioblastoma patients. Clin Cancer Res 16: 800-813, 2010. 